\title{
Une approche variationnelle de la mécanique du défaut
}

\author{
G.A. FRANCFORT \& J.J. MARIGO
}

Résumé. De nombreux micromécanismes font obstacle au comportement monoconstitutif de la plupart des matériaux élastiques. Leur prise en compte macroscopique est l'objet de la mécanique des défauts qui vient compléter la description constitutive du comportement par l'ajout de variables supplémentaires. Ainsi en est-il traditionnellement de la mécanique de l'endommagement et de celle de la rupture, ce dans le cadre fragile.

Dans les deux situations les méthodes classiques utilisées par les mécaniciens se révèlent, malgré leurs succès, déficientes sur plusieurs points. Nous proposons une attitude quelque peu différente qui consiste à adopter un principe de minimisation globale de la somme des énergies potentielles et des énergies dissipées par la création du défaut, analysons les aspects mathématiques de ce modèle ainsi que la facilité d'implémentation numérique et montrons en quoi il permet de remédier à certaines des pathologies associées aux méthodes classiques.

Mots clés. calcul des variations, élasticité, endommagement, espaces $B V$, homogénéisation, rupture fragile

\begin{abstract}
The presence of a variety of micromechanisms complexifies the constitutive behaviour of many elastic materials. Macroscopically, those materialize as defects, the description of which necessitates the introduction of additional internal variables. Such is the case of brittle damage and brittle fracture.

In both settings, the classical methods used by mechanicians exhibit, in spite of their achievements, quite a few shortcomings. Our standpoint is a bit at odds with the classical one. We propose a global minimization principle for the sum of the potential energy and of that dissipated by the growth of the defect. We then analyze its mathematical features, as well as its numerical feasibility and show how it allows for a partial removal of the usual pathologies of the classical methods.
\end{abstract}

Keywords. brittle fracture, $B V$ spaces, calculus of variations, damage, elasticity, homogenization

AMS Subject Classification. 35L05, 35T 


\section{Introduction}

Le comportement des matériaux élastiques est sérieusement affecté par la croissance ou/et la propagation de défauts qui peuvent entraîner leur affaiblissement et aboutir à leur destruction. Une analyse cohérente de ces phénomènes implique sans doute une descente à l'échelle interatomique : ainsi dans un cadre de mécanique classique pourrait-on songer à introduire des potentiels d'interaction entre atomes qui exhiberaient et des interactions entre non-voisins et des supports finis; il conviendrait alors de passer d'un tel modèle discret à son analogue continu, ce par un processus de moyennisation dont les modalités ne sont pas encore bien comprises. C'est là le chemin qui est suggéré par les travaux de e.g. [40]; un processus de "moyennisation" monodimensionnel est démontré dans [18]. Il semble cependant que ces exemples soient pour l'instant isolés et une démarche plus générale reste hors du champ actuel de connaissances, sauf peut-être en recourant massivement à la simulation numérique, si l'on croit que $10^{7}$ particules simulent raisonnablement le comportement macroscopique d'un solide.

Le palliatif du mécanicien est alors de postuler une phénoménologie qui va, si elle est adaptée, reproduire plus ou moins bien celle observée. En matière de rupture, le paradigme du défaut, le modélisateur, A. GRIFFiTh, se révéla brillant et son approche reste, soixante ans plus tard, la plus utilisée par les mécaniciens. Il n'en est pas de même d'autres phénomènes de défaut : preuve en est le désordre conceptuel qui règne en mécanique de l'endommagement (voir à ce sujet notre introduction dans [28]).

Dans cet article, nous nous limitons aux deux types de défauts précédemment évoqués, rupture et endommagement. Une approche plus ambitieuse devrait sans doute englober bien d'autres types de défauts, cavitation, décohésion dans les composites, et pourquoi pas plasticité qui du point de vue interatomique mentionné plus haut ne devrait pas se distinguer conceptuellement de la rupture, le matériau devant choisir, à l'aide du potentiel d'interaction s'il préfère rompre une liaison ou échanger une liaison par glissement sur un "plan cristallin". Nous adoptons une attitude phénoménologique, faute de mieux, mais nous la voulons minimaliste. Il s'agit d'introduire aussi peu de postulats ou paramètres que possible tout en s'efforçant de décrire et de prédire l'apparition et la progression des défauts étudiés de manière réaliste.

Les résultats présentés ici sont le fruit d'un effort collectif dans plusieurs domaines et avec plusieurs collaborateurs : nous citerons ici tout particulièrement B. Bourdin qui a développé toute l'implémentation numérique du modèle de rupture et I. Fonseca qui a grandement collaboré aux aspects mathématiques de la modélisation proposée.

Dans une première section (Section 2), nous présenterons les théories classiques de la rupture et de l'endommagement, en évoquant brièvement leurs forces et faiblesses. En Section 3, nous présenterons rapidement notre approche qui se veut résolument variationnelle. La Section 4 sera consacrée au cas de l'endommagement et la Section 5 au cas de la rupture. Nous tenterons de convaincre que, au prix d'un postulat- certes arbitraire, mais non en désaccord avec les canons de la thermodynamique rationnelle - de minimi-

sation à chaque instant de l'énergie totale, soit de la somme de l'énergie potentielle et de l'énergie associée au défaut (énergie de surface en rupture, et de volume en endommage- 
ment), nous sommes à même de fournir des prédictions quantitatives de l'apparition et de la propagation du défaut, ce même dans des contextes où les théories classiques avouent leur impuissance. Enfin la Section 6 sera consacrée à une brève description des défauts et insuffisances de la méthode proposée.

Cette analyse, qui est restreinte aux évolutions quasistatiques ne nous satisfait cependant pas : d'une part la modélisation proposée est, elle aussi, confrontée à des obstacles non résolus à ce jour (voir par exemple le problème des forces en rupture dans la Section 6); d'autre part, une bonne modélisation — et c'est là une évidence qu'on tait trop souvent se doit de considérer la dynamique. L'évolution quasistatique, si elle l'est vraiment, se lira sur les solutions du problème dynamique. On entre de ce fait dans le domaine des systèmes hyperboliques ... Un compromis serait de renoncer à un principe de minimisation globale et d'essayer d'analyser les minima locaux, voire les points stationnaires de l'énergie totale.

\section{Théories classiques du défaut}

Dans cette section nous examinons rapidement les modélisations classiques de l'endommagement et de la rupture dans un cadre quasistatique, sans prétention exhaustive. Dans ces deux contextes, on supposera que le matériau se comporte élastiquement et que les déformations restent petites, i.e., que le tenseur de GREEN-LAGRANGE $e(u)$ se réduit à

$$
e(u)=1 / 2\left(\nabla u+\nabla u^{t}\right)
$$

où $u$ dénote le champ de déplacement.

Schématiquement, l'esprit de la modélisation classique d'un mécanisme de défaut est toujours le même. On introduit un paramètre $\beta$ qui est censé caractériser le défaut. Il peut être à valeur scalaire ou tensorielle, même être une fonction. On calcule l'énergie potentielle comme fonction du temps $t$, du champ de déplacement $u$ et de $\beta$, soit $P(u, \beta, t)$. On la minimise tout d'abord à $t$ fixe (hypothèse de quasistaticité), mais aussi à $\beta$ fixe, afin d'obtenir le champ de déplacement $u(\beta, t)$ à l'équilibre, à cet instant, pour cet état de défaut. Enfin on détermine la valeur $\beta(t)$ que prendra le paramètre de défaut à l'instant t à l'aide soit d'un critère $f(t, e(u(\beta(t), t)), \beta(t)) \leq 0$ soit d'une loi d'évolution $\frac{d \beta}{d t}(t)=$ $g(t, e(u(\beta(t), t)), \beta(t))$. Examinons cette démarche plus en détail pour l'endommagement et la rupture.

\subsection{Endommagement}

L'endommagement décrit l'apparition et l'évolution de zones à rigidité affaiblie, celles-ci pouvant résulter d'une variété de "microphénomènes" dont l'étude précise est considérée a priori comme hors de portée du fait de leur complexité apparente et dont en revanche on prend le parti d'en rendre compte de façon "effective". Les modèles d'endommagement suivent généralement la démarche décrite ci-dessous [33]. Le paramètre $\beta$ est joué par un paramètre d'endommagement scalaire $\alpha$ caractérisant le degré d'affaiblissement du matériau. Ce paramètre sera soit bivalué $(0,1)$, c'est l'endommagement brutal, soit continu 
$(\in[0,1])$, auquel cas on parle d'endommagement progressif. Dans les deux cas, il faut se donner a priori la valeur de la rigidité - du tenseur d'élasticité dénoté par $A$ - en fonction de $\alpha$. Dans le cadre brutal on associe à chacune des valeurs de $\alpha$ une valeur de $A$, soit $A_{s}$ (s pour sain) et $A_{e}$ (e pour endommagé), avec $A_{e} \leq A_{s}$, donc, au point $x$ et à l'instant $t, A(x, t)=\alpha(x, t) A_{e}+(1-\alpha(x, t)) A_{s}$. Dans le cadre progressif $A(x, t)$ est une fonction, décroissante au sens des formes quadratiques, de $\alpha(x, t)$, soit $A(x, t)=A(\alpha(x, t))$.

De plus, on a besoin d'un critère pour déterminer la valeur de $\alpha$ au point et à l'instant considéré. Ce critère conditionne l'état d'endommagement à l'état de déformation considéré. Il est sous la forme d'une inégalité $f(t, e(u)(x, t)) \leq 0$ régissant le passage de l'état sain à l'état endommagé dans le cas brutal, alors que, dans le cadre progressif, il se présente généralement sous la forme d'une loi d'évolution, $\frac{d \alpha}{d t}(x, t)=g(t, e(u)(x, t), \alpha(x, t))$.

On voit donc que pour espérer une théorie predictive dans ce contexte, il faut deux types d'ingrédients : la dépendance fonctionnelle de $A$ par rapport à $\alpha$, et le critère. De plus le caractère local de cette description conduit à des lois d'endommagement indépendantes de la taille de l'échantillon ou de la forme des sollicitations appliquées, ce qui est en contradiction avec l'expérience qui constate, elle, un effet dit d'échelle.

\section{$2.2 \quad$ Rupture}

La rupture décrit l'apparition et l'évolution d'une ou de plusieurs fissures, i.e., de surfaces libres de codimension 1 à l'intérieur du matériau. Les modèles de rupture suivent généralement la démarche proposée par GRIFFITH et décrite ci-dessous dans le cas bidimensionnel [30], [19].

On suppose a priori connu le trajet de fissuration, soit $\Gamma_{\text {tot. }}$, une courbe dans le domaine $\Omega$ occupé par le matériau - courbe qui, si on ne se la donnait pas, jouerait le rôle $\mathrm{du}$ paramètre $\beta$ caractérisant le défaut. Pour une fissure $\Gamma \subset \Gamma_{\text {tot. }}$ de longueur $l-$ longueur que l'on va donc pouvoir prendre comme paramètre $\beta$-, à l'instant $t$, on calcule l'énergie potentielle associée, pour le chargement appliqué à cet instant, qu'on supposera être un déplacement imposé $U(x, t)$ sur $\partial \Omega$, i.e.,

$$
E_{d}(l, t)=\min _{u=U(t) \text { sur } \partial \Omega} \int_{\Omega \backslash \Gamma} 1 / 2 A e(u) \cdot e(u) d x .
$$

Puis, en supposant la régularité adéquate de $E_{d}$ par rapport à $l$, on calcule le taux de restitution d'énergie associé à la fissure effective, soit $\Gamma(t)$ de longueur $l(t)$,

$$
G(t)=-\frac{\partial E_{d}}{\partial l}(l(t), t)
$$

La loi d'évolution proposée par GRIfFith est alors la suivante :

- $\frac{d l}{d t}(t) \geq 0$ (la fissure ne peut que grandir),

- $G(t) \leq k$ ( $k$ est un taux critique de restitution d'énergie),

- $\frac{d l}{d t}(t)(G(t)-k)=0$ (la fissure grandit quand le taux de restitution est critique), 
l'inégalité $G(t) \leq k$ constituant le critère de GRIFFITH proprement dit.

Ce critère, malgré son endurance, souffre de trois faiblesses principales illustrées dans [28], [29]. D'abord, le trajet de fissuration ne peut pas être déterminé si on ne se le donne pas a priori, car le critère ne fournit qu'une équation pour les deux inconnues que sont alors les coordonnées de la pointe de fissure. Ensuite, l'initiation de la fissuration est impossible si $l(0)=\frac{\partial E_{d}}{\partial l}(0, \cdot)=0$. Enfin, une croissance régulière de $\Gamma(t)$ est impossible si $E_{d}(l(t)$, temps d'initiation) est une fonction concave de $t$.

Pour pallier à ces insuffisances, certains ajouts ont été proposés. Dans le cas d'une fissure tournante, des critères supplémentaires sont introduits pour déterminer l'angle de rotation (cf. [10]); pour ce qui est de l'initiation, on suppose généralement la préexistence d'une entaille (crack notch); le défaut de régularité conduit quelquefois à l'introduction d'un critère généralisé [31], alors que, dans le cas de figure évoqué plus haut, on juge de la stabilité de la fissuration par un critère aux dérivées secondes [38]. Bref, c'est là du cas par cas.

\section{Une approche variationnelle}

L'approche que nous suggérons est a priori très proche de l'approche classique. Si l'on reprend le formalisme introduit au début de la Section 2, on se contente de substituer au critère, ou à la loi d'évolution, une énergie dissipée $K(u, \beta)$ par la croissance du défaut et de postuler que le couple $(u, \beta)$ solution de l'évolution devra minimiser l'énergie totale $P+K$ parmi tous les $u$ cinématiquement admissibles et $\beta$ conformes aux états de défaut antérieurs. Ce postulat de minimisation n'est pas étranger à la modélisation de phénomènes irréversibles en mécanique des milieux continus : citons par exemple le principe de dissipation plastique maximale, ou celui de dissipation visqueuse maximale pour un mélange de fluides immiscibles; il ne découle pourtant pas de principes thermodynamiques connus - mais n'en contredit aucun — et n'est sans doute qu'une piètre approximation du comportement réel ainsi qu'on l'a remarqué plus haut.

Les atouts de cette approche sont plus subtils. Comme nous le démontrerons plus loin, un tel principe permet de s'affranchir d'une phénoménologie redondante pour ce qui est de l'endommagement et d'affiner la description type Griffith pour ce qui est de la rupture.

Décrivons maintenant plus précisément le modèle dans ces deux cadres. On supposera que le matériau occupe un domaine $\Omega$ et qu'il est soumis à un déplacement imposé $U$ (fonction de $(x, t))$ sur une partie $\partial \Omega_{d}$ de sa frontière. De plus, on suppose le matériau en équilibre à chaque instant $t$.

1. Endommagement : On va considérer un modèle de type brutal avec deux états caractérisés par leur densités d'énergie respectives, $W^{s}(e) \geq W^{e}(e)$. On n'a pas besoin ici de se spécialiser au cas linéaire où $W^{s}(e)=1 / 2 A_{s} e \cdot e$ et $W^{e}(e)=1 / 2 A_{e} e \cdot e$. On dénote par $\chi$ la fonction caractéristique de la zone endom magée et donc l'énergie 
potentielle associée à un champ cinématiquement admissible $u$ à l'instant $t$ est

$$
P(u, \chi, t)=\int_{\Omega}\left(\chi(x) W^{e}+(1-\chi(x)) W^{s}\right)(e(u)(x)) d x .
$$

On introduit, dans l'esprit de GRIfFITH l'énergie dissipée suivante :

$$
K(\chi)=\int_{\Omega} \chi(x) d x
$$

2. Rupture: On va ici se permettre n`importe quel type de fissure, tant dans le domaine que sur son bord. Le paramètre $\beta$ est alors très complexe puisqu'il s'agit de tous les fermés $\Gamma$ de $\bar{\Omega}$. Un champ cinématiquement admissible $u$ à l'instant $t$, et pour un $\Gamma$ donné, est un champ qui vaut $U$ sur $\partial \Omega_{d} \backslash \Gamma-\Gamma$ peut "manger" une partie de $\partial \Omega_{d}$ — et l'énergie potentielle associée est

$$
P(u, \Gamma, t)=\int_{\Omega \backslash \Gamma} W(e(u)(x)) d x,
$$

où $W$ dénote la densité d'énergie élastique (une fois encore pas nécessairement quadratique). Conformément à la théorie de GRIfFith, l'énergie dissipée est donnée par

$$
K(\Gamma)=k \mathcal{H}^{N-1}\left(\Gamma \cap\left(\Omega \cup \partial \Omega_{d}\right)\right),
$$

où $\mathcal{H}^{N-1}$ dénote la mesure de Hausdoorf $N$ - 1-dimensionnelle. Remarquons que, comme on va minimiser la somme $P+K$, on éliminera automatiquement les $\Gamma$ trop gros, i.e., tels que $\mathcal{H}^{N-1}(\Gamma)=+\infty$.

Remarque 3.1 Nous attirons l'attention du lecteur sur la forme précise de l'énergie de surface; l'ensemble $\Gamma \cap\left(\Omega \cup \partial \Omega_{d}\right)$ pour lequel on paie de l'énergie de surface est, nous semble-t-il, le bon. On doit en effet compter l'énergie de surface dissipée pour créer une fissure, et à l'intérieur du domaine, et sur la partie de la frontière où le déplacement est imposé, mais pas sur celle qui est libre.

Dans ces deux cadres, on se propose de minimiser, à l'instant $t, P+K$ par rapport à $(\chi, u)$, resp. $(\Gamma, u)$, ce sous deux contraintes :

1. Irréversibilité : Le défaut ne peut que croître, ce qui conduit à n'envisager que des défauts $\chi$ ou $\Gamma$ tels que

$$
\left\{\begin{array}{l}
\left.\chi \geq \sup _{s<t} \chi(s) \text { (en tant que fonction de } x\right) \\
\Gamma \supset \cup_{s<t} \Gamma(s)
\end{array}\right.
$$

$\chi(s)$ et $\Gamma(s)$ désignant les valeurs que prendront effectivement les défauts à l'instant $s$. 
2. Mémoire : Cette deuxième condition, qui peut sembler surprenante, est dictée par un passage d'un temps discret à un temps continu (cf. [28]). Elle traduit une sorte d'effet de mémoire des états de défaut précédents, soit

$$
\left\{\begin{array}{l}
\inf _{u} P(u, \chi(t), t)+K(\chi(t)) \leq \inf _{s<t}\left\{\inf _{u} P(u, \chi(s), t)+K(\chi(s))\right\} \\
\inf _{u} P(u, \Gamma(t), t)+K(\Gamma(t)) \leq \inf _{s<t}\left\{\inf _{u} P(u, \Gamma(s), t)+K(\Gamma(s))\right\}
\end{array} .\right.
$$

Remarque 3.2 Nous renvoyons le lecteur à [28] pour une analyse plus détaillée de la deuxième condition ci-dessus. Notons que cette condition disparaît lorsqu'on discrétise le temps.

Nous ne sommes pas, à ce jour en mesure d'analyser de manière mathématiquement satisfaisante les deux problèmes d'évolution ainsi formulés. Cependant, les remarques suivantes montrent la richesse (et la complexité) potentielle de ces formulations.

Remarque 3.3 Dans le cadre de l'endommagement, on verra dans la Section \& que, pour un chargement indépendant de $t$, le problème, qui devient indépendant de $t$, n'a pas de solution et qu'on doit le relaxer. Cette tâche, que l'on mène à bien dans ce cas, reste à accomplir dans le cas d'évolution, pour lequel on se contente de postuler une formulation relaxée, qu'on sait d'ailleurs ne pas être la bonne (cf. Remarque 4.3 plus loin).

Remarque 3.4 Dans le cadre de la rupture, il est formellement montré dans [28] que, dans le cas d'un chargement de la forme $U(x, t)=t U(x)$, qu'on appellera un chargement monotone, le modèle prédit l'initiation de la rupture en temps fini et la destruction de la structure. Précisément, si $\Gamma(0)=\emptyset$, il existe $t_{0}$ tel que $\Gamma(t) \neq \emptyset, t>t_{0}$, et il existe $t_{f}$ tel que $\inf _{u} P(u, \Gamma(t), t)=0, t>t_{f}$. De plus, $t_{f} \geq t_{i}$, et on peut avoir $t_{f}=t_{i}$.

On se propose maintenant de temporellement discrétiser l'évolution, ce qui est, en tout état de cause, ce que tout calcul effectue. On n'a pas alors à considérer la deuxième contrainte car on se persuade immédiatement qu'elle est discrètement satisfaite si la version discrétisée de la première, proposée ci-dessous, l'est, en tout cas pour des chargements de type monotones qui est la classe restreinte de chargements dorénavant considérée. On regarde donc une suite de temps $t_{0}=0 \leq t_{1} \leq \ldots \leq t_{P}=T$, avec $U_{0}=0$ et $\chi_{0}=0\left(\right.$ resp. $\left.\Gamma_{0}=\emptyset\right)$, le problème au temps $t_{i}$ devenant:

$\mathcal{P}_{i}: \quad \quad \quad$ Minimiser $P\left(u, \chi(\right.$ resp. $\left.\Gamma), t_{i}\right)+K(\chi($ resp. $\Gamma))$ parmi tous les champs $(u, \chi)$ tels que $u=U_{i}$ sur $\partial \Omega_{d}$, resp. $(u, \Gamma)$ tels que $u=U_{i}$ sur $\partial \Omega_{d} \backslash \Gamma$, ce sous la contrainte $\chi \geq \chi_{i-1}$, resp. $\Gamma \supset \Gamma_{i-1}$.

Nous allons examiner le problème $\mathcal{P}_{i}$ dans les cas de l'endommagement (Section 4) et de la rupture (Section 5). 


\section{L'endommagement}

Dans une première sous-section, nous analysons le premier pas de temps $t_{1}$ lors d'un chargement $U\left(t_{i}, x\right)$. Le problème de minimisation à cet instant n'a pas de solution et on doit le relaxer. On ne sait plus alors comment imposer, aux pas de temps suivants la contrainte d'irréversibilité $\chi \geq \chi_{i-1}$ et ceci nous conduit dans une deuxième section à introduire pour les temps suivants une évolution dont on ne sait affirmer qu'elle est une vraie relaxation du problème de départ. Voir à ce sujet la Remarque 4.3.

Dans ce qui suit, on va supposer que $W^{s}, W^{e}$ sont des fonctions continues de $\mathbb{R}^{N^{2}}$ dans $\mathbb{R}$ telles que

$$
\kappa|\xi|^{p} \leq W^{s}(\xi), W^{e}(\xi) \leq \gamma\left(1+|\xi|^{p}\right),
$$

où $\kappa, \gamma$ sont des nombres positifs et $1<p<+\infty$.

\subsection{Le premier pas de temps}

On s’intéresse donc au temps $t_{1}$, pour lequel le chargement est $U_{1}$ sur $\partial \Omega_{d}$. Le problème $\mathcal{P}_{1}$ devient

$$
\begin{aligned}
& I_{1} \equiv \inf _{u}\left\{\int_{\Omega} \Psi_{1}(e(u)(x)) d x, u \in W^{1, p}\left(\Omega ; \mathbb{R}^{N}\right) ; u=U_{1} \text { sur } \partial \Omega_{d}\right\}, \\
& \Psi_{1}(\xi) \equiv \inf _{\chi \in\{0,1\}}\left\{\left(\chi W^{e}+(1-\chi) W^{s}\right)(\xi)+k \chi\right\} .
\end{aligned}
$$

L'intégrand $\Psi_{1}$ n'est pas nécessairement quasiconvexe et $I_{1}$ n'est donc pas nécessairement atteint. On doit quasiconvexifier $\Psi_{1}$ (cf. [1]). On obtient

$$
I_{1}=\min _{u}\left\{\int_{\Omega} Q\left[\Psi_{1}\right](e(u)(x)) d x, u \in W^{1, p}\left(\Omega ; \mathbb{R}^{N}\right) ; u=U_{1} \text { sur } \partial \Omega_{d}\right\},
$$

où $Q\left[\Psi_{1}\right]$, la quasiconvexifiée de $\Psi_{1}$ est donnée par

$$
Q\left[\Psi_{1}\right](e)=\inf _{\phi}\left\{\int_{Y} \Psi_{1}(e+e(\phi)(y)) d y, \phi \in W_{p e r}^{1, p}\left(Y ; \mathbb{R}^{N}\right)\right\}
$$

Dans l'expression précédente, $Y$ est l'hypercube unité de $\mathbb{R}^{N}$ et $W_{p e r}^{1, p}\left(Y ; \mathbb{R}^{N}\right)$ les fonctions de $W^{1, p}\left(Y ; \mathbb{R}^{N}\right)$ à trace périodique.

Remarque 4.1 On a implicitement étendu dans ce qui précède la notion de fonction quasiconvexe et de quasiconvexifiée au cas des gradients symétrisés qui n'est pas l'objet de [1]. Les résultats obtenus dans [1] restent identiques dans le contexte des gradients symétrisés. Nous ne connaissons pas, à ce jour, de résultats précis dans la littérature qui traitent de la quasiconvexification de telles fonctionnelles, bien que la formule de quasiconvexification soit largement employée. En ce qui concerne la notion de quasiconvexité pour des fonctionnelles qui dépendent du gradient symétrisé, on peut se référer à [26] qui traite de manière générale de la notion de quasiconvexité pour des fonctionnelles dépendant de champs qui satisfont à des systèmes différentiels à coefficients constants (c'est le cadre de 
la compacité par compensation), ce qui est le cas pour les gradients symétrisés qui satisfont les équations de compatibilité $e_{i j, k h}+e_{k h, i j}-2 e_{i k, j h}=0$.

Il faut de plus se servir du Théorème 3.1, Corollaire 3.2 et Conjecture 3.7(2) dans [11] ou de l'équation (2.12) et du Lemme 2.2 dans [32] pour remplacer les conditions de DiRICHLET par des conditions périodiques.

Le calcul de $Q\left[\Psi_{1}\right]$ peut être affiné en permutant les infima en $\phi$ et $\chi$ comme suit :

$$
\begin{aligned}
Q\left[\Psi_{1}\right](e)=\inf _{\chi \in L^{\infty}(Y ;\{0,1\})} & \left\{\operatorname { i n f } _ { \phi \in W _ { p e r } ^ { 1 , p } ( Y ; \mathbb { R } ^ { N } ) } \left\{\int _ { Y } \left\{\left(\chi W^{e}+(1-\chi) W^{s}\right)(e+\right.\right.\right. \\
& e(\phi)(y))+k \chi(y)\} d y\}\} \\
=\inf _{0 \leq \theta \leq 1} & \{\Phi(e, \theta)+k \theta\},
\end{aligned}
$$

où

$$
\begin{aligned}
\Phi(e, \theta) & \equiv \inf _{\chi \in L^{\infty}(Y ;\{0,1\})}\left\{W^{h}(e, \chi), \int_{Y} \chi(y) d y=\theta\right\} \\
W^{h}(e, \chi) & \equiv \inf _{\phi \in W_{p e r}^{1, p}\left(Y ; \mathbb{R}^{N}\right)}\left\{\int_{Y}\left\{\left(\chi W^{e}+(1-\chi) W^{s}\right)(e+e(\phi)(y))\right\} d y\right\} .
\end{aligned}
$$

L'énergie $W^{h}(e, \chi)$ ressemble à l'énergie homogénéisée associée au mélange périodique de $W^{s}$ et de $W^{e}$ occupant respectivement dans $Y$ les domaines $\{\chi(y)=0\}$ et $\{\chi(y)=1\}$. Cette analogie est rigoureuse si $W^{s}$ et $W^{e}$ sont convexes [35]. Dans le cas contraire, ceci n'est pas l'expression de l'énergie homogénéisée [37]. Cependant, $\Phi(e, \theta)$ est toujours l'infimum sur tous les mélanges périodiques à proportion $\theta$ donnée de $W^{s}$ et de $W^{e}$ (cf. [25], Remark 3.8). Dans le language de l'homogénéisation, ceci est un problème de bornes sur les énergies effectives de biphasés. On ne sait, à ce jour, traiter que le cas quadratique, i.e., $W^{s}\left(\operatorname{resp} . W^{e}\right)(\xi)=1 / 2 A_{s}\left(\operatorname{resp} . A_{e}\right) \xi \cdot \xi$; cf. [4], [22], et aussi [27] dans le cas bidimensionnel. Il reste alors à minimiser $\Phi(e, \theta)+k \theta$ pour $\theta \in[0,1]$; c'est une procédure algébrique, mais en général inextricable analytiquement. A titre d'exemple, nous reproduisons ci-après, dans le cas quadratique et pour des élasticités isotropes, i.e.,

$$
\left(A_{s}\right)_{i j k h}\left(\operatorname{resp} .\left(A_{e}\right)_{i j k h}\right)=\lambda_{s}\left(\operatorname{resp} . \lambda_{e}\right) \delta_{i j} \delta_{k h}+\mu_{s}\left(\operatorname{resp} . \mu_{e}\right)\left(\delta_{i k} \delta_{j h}+\delta_{i h} \delta_{j k}\right),
$$

l'expression de $Q\left[\Psi_{1}\right]\left(a i_{2}\right)$, où $i_{2}$ dénote la matrice identité sur $\mathbb{R}^{2}$ et $a$ est un scalaire. On obtient (cf. [27], Proposition 3.3) :

$$
Q\left[\Psi_{1}\right]\left(a i_{2}\right)= \begin{cases}2\left(\lambda_{s}+\mu_{s}\right) a^{2}, & \text { si }|a| \leq \frac{a_{c}}{q}, \\ 2 \frac{\lambda_{s}+\mu_{s}}{q^{2}-1}\left\{2 p q a_{c}|a|-p a_{c}^{2}-\left(1-(1-p) q^{2}\right) a^{2}\right\}, & \text { si } \frac{a_{c}}{q} \leq|a| \leq q a_{c} \\ 2\left(\lambda_{e}+\mu_{e}\right) a^{2}+k, & \text { si }|a| \geq q a_{c},\end{cases}
$$

où $p \equiv 1-\frac{\lambda_{e}+\mu_{e}}{\lambda_{s}+\mu_{s}}, q \equiv \sqrt{\frac{\lambda_{s}+\mu_{s}+\mu_{e}}{\lambda_{e}+2 \mu_{e}}}, a_{c} \equiv \sqrt{k /\left(2 p\left(\lambda_{s}+\mu_{s}\right)\right)}$. Dans ce cas particulier, on vérifie aisément que la densité d'énergie n'est pas convexe en $a$ ! 
Remarque 4.2 Dans le cas particulier évoqué ci-dessus, la fonction $\Phi(e, \theta)$ est, pour un e donné, strictement convexe en $\theta$; il y a donc un unique $\theta$ associé à $Q\left[\Psi_{1}\right](e)$, pour tout $e$; c'est la fraction volumique de matériau endommagé.

Au vu de la remarque précédente, le modèle relaxé obtenu au premier pas de temps s'apparente à un modèle d'endommagement progressif. Alors qu'on est parti de deux états - le sain et l'endommagé - , on arrive, par cette relaxation, à un contin$11 u m$ d'états paramétrés notamment par la fraction volumique $\theta_{1}(x)$ associée à la solution $u_{1}(x)$ du problème de minimisation de $\int_{\Omega} Q\left[\Psi_{1}\right](e(u(x)) d x$ avec la condition aux limites $u=U_{1}$ sur $\partial \Omega_{d}$. De plus pour ce $\theta_{1}(x)$, la ou les microstructures possibles doivent minimiser $W^{h}\left(e\left(u_{1}\right)(x), \chi\right)$ parmi tous les $\chi(y)$ définis sur $Y$ tels que $\int_{Y} \chi(y) d y=\theta_{1}(x)$. En d'autres termes les microstructures possibles sont déterminées a posteriori et non postulées a priori comme c'est le cas dans nombreux modèles d'endommagement par micromécanisme (cf. e.g. [34]). Enfin le caractère non local de l'évolution de l'endommagement est pris en compte puisque la solution $u_{1}$ et donc à leur tour $\theta_{1}$ et les microstructures optimales dépendent et de la géométrie et du chargement.

\subsection{Les pas de temps suivants}

Comme le premier pas de temps a nécessité la relaxation de la fonctionnelle de départ, on n'a plus, à l'issu de ce premier chargement de zone endommagée bien définie - plus de $\chi_{1}$ - et on ne sait comment imposer la contrainte d'irréversibilité discrétisée $\chi_{2} \geq$ $\chi_{1}$. C'est pourquoi nous postulons une contrainte d'irréversibilité affaiblie ainsi qu'une formulation "relaxée" du problème. Plus précisément, nous postulons qu'au pas de temps suivants la fonctionnelle à minimiser est

$$
\int_{\Omega} Q \Psi_{i}(x, e(u)(x)) d x
$$

où

$$
Q \Psi_{i}(x, e) \equiv \inf _{\theta_{i-1}(x) \leq \theta \leq 1}\{\Phi(e, \theta)+k \theta\} .
$$

En d'autres termes, nous avons affaibli l'irréversibilité en imposant seulement $\theta(x) \geq$ $\theta_{i-1}(x)$. Dans [25], Section 3, la quasiconvexité de $Q \Psi_{i}(x, e)$ est discutée. Elle est certainement satisfaite dans le cas quadratique. Le cas d'énergies $W_{s}$ ou $W_{d}$ non convexes reste ouvert. Si on admet cette propriété, alors on peut continuer l'évolution aux pas de temps suivants. Notons que l'unicité des $u_{i}$ n'est pas assurée, et donc qu'il peut y avoir bifurcation des solutions à chaque pas de temps.

Remarque 4.3 La formulation "relaxée" pour les pas de temps suivants est incomplète. En effet, une vraie relaxation suppose qu'on considère d'un coup les P-pas de temps et $q u$ 'on examine les $P$ suites minimisantes $\chi_{1 n} \leq \ldots \leq \chi_{P_{n}}$ de fonctions caractéristiques pour les $P$ problèmes $\mathcal{P}_{1}, \ldots, \mathcal{P}_{P}$. Comme l'homogénéisation conserve l'ordre, la contrainte de monotonie des suites $\chi_{i}$ va résulter en particulier en une contrainte de monotonie sur 
les énergies $W^{h}\left(e, \chi_{i}\right)$. Dans le cas quadratique par exemple, il faudrait donc au moins pouvoir calculer au temps $t_{2}$

$$
\inf _{A_{h}(\chi) \leq A_{h}\left(\chi_{1}\right)}\left\{1 / 2 A_{h}(\chi) e \cdot e ; \int_{Y} \chi(y) d y=\theta\right\},
$$

où $\chi_{1}$ est une microstructure qui atteint la borne $W^{h}\left(e, \theta_{1}\right)$ et où $A_{h}(\chi)$ est le tenseur d'élasticité homogénéisé associé à $\chi$, ce qu'on ne sait pas faire.

Remarque 4.4 La formulation "relaxé" proposée conduit à des schémas numériques type directions alternées. En effet, on remarque que minimiser $\int_{\Omega} Q \Psi_{i}(x, e(u)(x)) d x$ en $u$ revient à minimiser $\int_{\Omega}\left\{W^{h}(e(u(x)), \chi)+k \theta(x)\right\} d x$ en $u, \chi, \theta$ avec $\int_{Y} \chi(y) d y=\theta(x)$, $\theta_{i-1}(x) \leq \theta(x) \leq 1$. On peut donc alterner entre une minimisation en $u \grave{a} \theta, \chi$ fixes, et une minimisation en $\theta, \chi \grave{a}$ u fixe. Mais cette deuxième minimisation est locale, i.e., il suffit de calculer $\inf _{\theta_{i-1}(x)<\theta<1}\{\Phi(e(u(x)), \theta)+k \theta\}$, ce qu'on sait faire explicitement dans le cas quadratique (cf. [2]). C'est là la base des calculs présentés dans [3] et dans [39].

Les résultats de calcul montrent une évolution des zones endommagées qui correspond aux zones à fortes contraintes.

\section{La rupture}

Dans une première sous-section, on examine le cas d'un problème antiplan pour lequel l'analyse mathématique est assez satisfaisante. Le problème admet une formulation faible dans l'espace $S B V(\Omega)$ des fonctions spéciales à variations bornées qui seront définies plus loin. Cette formulation est du même type que celle obtenue par De Giorgi, Carriero \& Leaci à partir de la fonctionnelle de Mumford \& Shah [23]. Le problème faible ne se prête pas à l'implémentation numérique parce que les fonctions test peuvent être discontinues. Un schéma d'approximation très proche de celui proposé par Ambrosio \& Tortorelli [7], [8] est exposé dans la Sous-section 2. D'autres approximations peuvent aussi être utilisées (cf. Remarque 5.3). La Sous-section 3 évoque le cas plan — ou plus généralement le cas tridimensionnel - pour lequel les résultats mathématiques sont au mieux partiels.

Dans cette section, on suppose que la densité d'énergie élastique est quadratique, i.e., de la forme

$$
W(\xi)=1 / 2 A \xi \cdot \xi,
$$

où $A_{i j k h} \xi_{i j} \xi_{k h} \geq \gamma|\xi|^{2}$, pour tout $\xi$ symétrique .

Remarque 5.1 Certains des résultats évoqués ci-dessous seraient encore vrais si la densité d'énergie était plus générale, mais l'équivalence entre formulation d'origine et formulation faible détaillée dans la Sous-section 1 est peut-être en défaut dans ce cas. C'est un problème ouvert, même dans le cas antiplan, si $W(\xi)$ n'est pas homogène en $\xi$.

On dénote par $\mathcal{N}_{1}$ le complémentaire de $\partial \Omega_{d}$ dans $\partial \Omega$. 


\subsection{Le cas antiplan}

On suppose donc ici que le problème est antiplan, i.e., que $N=2, \Omega \subset \mathbb{R}^{2}$ et $u=u(x, y) \mathbf{e}_{\mathbf{z}}$. Au premier pas de temps, on cherche donc à résoudre $\mathcal{P}_{1}$, soit à minimiser

$$
\int_{\Omega \backslash \Gamma} 1 / 2|\nabla u|^{2} d x+k \mathcal{H}^{1}\left(\Gamma \backslash \mathcal{N}_{1}\right)
$$

parmi tous les champs $(u, \Gamma)$ tels que $\Gamma$ (fermé $) \subset \bar{\Omega}$ et $u \in W^{1,2}(\Omega \backslash \Gamma) ; u=U_{1}$ sur $\partial \Omega_{d} \backslash \Gamma$ (la trace de la fonction $u$ sur $\partial \Omega_{d} \backslash \Gamma$ doit être bien comprise ; voir à ce propos la formulation du problème $\mathcal{P}_{1}$-faible ci-dessous). Ce problème n'est pas facilement topologisable à cause de la minimisation en $\Gamma$ et on souhaiterait une reformulation dans un espace fonctionnel plus "familier". C'est là, dans le cadre proche de la segmentation d'images, l'objet de [23]. Il s'agit dans ce dernier cadre de minimiser, pour $\Gamma$ (fermé ) $\subset \bar{\Omega}$ et $u \in W^{1,2}(\Omega \backslash \Gamma)$,

$$
\int_{\Omega \backslash \Gamma}|\nabla u|^{2} d x+k \mathcal{H}^{N-1}(\Gamma \cap \Omega)+k^{\prime} \int_{\Omega}|u-g|^{2} d x
$$

où $u$ est l'intensité de pixels recherchée (l'image), g est celle observée (la photo), et $k, k^{\prime}$ sont des poids. Il n'y a pas alors de conditions aux limites sur $u$.

La formulation faible proposée dans [23] est la suivante:

Minimiser $E(u) \equiv \int_{\Omega}|\nabla u|^{2} d x+k \mathcal{H}^{N-1}\left(S_{u}\right)+k^{\prime} \int_{\Omega}|u-g|^{2} d x$, pour $u \in S B V(\Omega)$.

Ici, $S B V(\Omega)$ est le sous-espace des fonctions de $B V(\Omega)$ dont les dérivées (des mesures) n'ont pas de parties de CANTor, i.e., telles que

$$
D u=\nabla u \mathcal{L}^{N}+\mathcal{H}^{N-1} \mid S_{u},
$$

où $S_{u}$ dénote le complémentaire de l'ensemble des points de LeBEsgue de $u$ et $\nabla u$ la densité de $D u$ par rapport à $\mathcal{L}^{N}$. Il est montré dans [23] que $(u, \Gamma)$ est un minimum du problème d'origine si et seulement si $u$ est un minimum du problème faible; de plus, $\left(u, \overline{S_{u}}\right)$ est alors minimum du problème d'origine.

Dans le cadre de la rupture antiplane, on a la condition aux limites $u=U_{1}$ sur la partie $\partial \Omega_{d}$ du bord. Il faut alors adapter les arguments de [23] et ce de manière non triviale, car les fissures peuvent aller au bord de $\Omega$. Ceci est étudié dans [20] dans le cas $\partial \Omega_{d}=\partial \Omega$. Le cas où une partie seulement du bord est contrainte - le cas réaliste en mécanique - est étudié dans [16], Chapitre 1. Il s'agit alors du problème suivant:

Problème $\mathcal{P}_{1}$-faible :

Minimiser $\int_{\Omega}|\nabla u|^{2} d x+k \mathcal{H}^{1}\left(S_{u} \backslash \mathcal{N}_{1}\right)$, pour $u \in S B V(\hat{\Omega})$ avec $u=U_{1}$ sur $\hat{\Omega} \backslash \bar{\Omega}$.

Ici, $\hat{\Omega}$ est un domaine qui contient compactement $\Omega$ et la donnée aux limites $U_{1}$ - ou plutôt son prolongement à $\mathbb{R}^{2}$ — est supposé(e) Lipschitz. On suppose de plus que $\Omega$ a une frontière Lipschitz et $\mathcal{C}^{1}$ par morceaux.

Remarque 5.2 Des domaines plus singuliers peuvent être considérés, par exemple des domaines $\mathcal{C}^{1}$ entaillés (cf. [16], Section 1.2.6). 
On montre aisément, à l'aide des résultats de semi-continuité inférieure d'AmBrosio ([5], Théorème 3.1 , [6], Théorèmes 2.1 et 3.6 ) l'existence d'un minimum à $\mathcal{P}_{1}$-faible.

Le résultat critique, obtenu dans [16] (Proposition 1.2.7) par une adaptation des résultats de [20] est un résultat de régularitésur le saut associé à une solution du problème faible :

Tout minimum $u$ de $\mathcal{P}_{1}$-faible est tel que $\mathcal{H}^{1}\left(\left(\overline{S_{u} \backslash \mathcal{N}_{1}}\right) \backslash\left(S_{u} \backslash \mathcal{N}_{1}\right)\right)=0$.

A l'aide de ce résultat, on montre l'équivalence entre problème d'origine et formulation faible comme dans le cas de la segmentation d'images et on trouve une solution $u_{1}, \overline{S_{u_{1}} \backslash \mathcal{N}_{1}}$ du problème d'origine.

Aux pas de temps suivants, $i \geq 1$, on peut alors répéter cet argument, en prenant cette fois pour nouveau domaine $\Omega_{i} \equiv \Omega_{i-1} \backslash\left(\overline{S_{u_{i-1}} \backslash \mathcal{N}_{i-1}}\right)$, et pour nouveau bord libre $\mathcal{N}_{i} \equiv \mathcal{N}_{i-1} \cup\left(\overline{S_{u_{i-1}}} \cap \partial \Omega\right)$. Encore faut-il pour cela que $\Omega_{i}$ et $\mathcal{N}_{i}$ aient la régularité voulue : grâce à une analyse précise des ingrédients nécessaires à la démonstration du résultat précédent de régularité, dans l'esprit de la remarque 5.2 , on peut en effet réitérer et on obtient l'équivalence entre les formulations pour tous les pas de temps. Donc, la formulation faible aux pas de temps suivants devient

Problème $\mathcal{P}_{i}$-faible :

Minimiser $\int_{\Omega_{i}}|\nabla u|^{2} d x+k \mathcal{H}^{1}\left(S_{u} \backslash \mathcal{N}_{i}\right)$, pour $u \in S B V(\hat{\Omega})$ avec $u=U_{i}$ sur $\hat{\Omega} \backslash \bar{\Omega}$.

Notons que le problème $\mathcal{P}_{i}$-faible admet un minimum par un argument identique à celui utilisé pour $\mathcal{P}_{1}$-faible, et ce indépendamment de la régularité de $\partial \Omega_{i}$.

\subsection{Approximation}

En adoptant la formulation $\mathcal{P}_{i}$-faible obtenue dans la sous-section précédente, on s'est débarrassé de la minimisation en $\Gamma$ de la formulation d'origine, mais ce par le passage à un espace de fonctions discontinues. Le calcul numérique, par éléments finis par exemple, semble impossible, faute de connaitre a priori le locus de ces discontinuités. L'idée qui va permettre un calcul est de "régulariser" la fonctionnelle à minimiser par $\Gamma$-convergence. On va de fait "étaler" le saut par l'introduction d'une deuxième variable. Dans le cadre de la segmentation d'images, ceci est accompli par Ambrosio \& TorTorelli dans [7], [8] qui proposent plusieurs variantes autour de la fonctionnelle suivante :

$$
E_{c}(u, v) \equiv \int_{\Omega}\left\{\left(v^{2}+k_{c}\right)|\nabla u|^{2}+c|\nabla v|^{2}+\frac{(1-v)^{2}}{4 c}\right\} d x
$$

où $k_{c}<<c$. Alors ils montrent que $E_{c}(u, v) \Gamma\left(L^{2}(\Omega ; \mathbb{R} \times[0,1])\right)$-converge, quand $c \rightarrow 0$ vers

$$
E(u, v) \equiv\left\{\begin{array}{l}
\int_{\Omega}|\nabla u|^{2} d x+\mathcal{H}^{1}\left(S_{u}\right), \text { si } v=1 \text { p.p. } \\
+\infty, \text { sinon. }
\end{array}\right.
$$

Il convient d'adapter la fonctionnelle approchée au cas qui nous intéresse, ce qui est 
fait dans [15]. La fonctionnelle, au premier pas de temps par exemple, est donnée par

$$
E_{c}(u, v) \equiv\left\{\begin{array}{l}
\int_{\Omega}\left(v^{2}+k_{c}\right)|\nabla u|^{2} d x+\int_{\hat{\Omega} \backslash \mathcal{N}_{1}}\left\{c|\nabla v|^{2}+\frac{(1-v)^{2}}{4 c}\right\} d x, u=U_{1} \text { sur } \hat{\Omega} \backslash \bar{\Omega} \\
+\infty, \text { sinon }
\end{array}\right.
$$

Nous ne détaillons pas plus ici cette approximation mais renvoyons le lecteur à [15], Chap. 1, Section 1.3.

L'approximation proposée ci-dessus doit à son tour être discrétisée. Une méthode est, dans le cas de la segmentation d’images, élaborée dans [12]. Une apparentée, mais plus simple, est, dans ce même cadre, étudiée dans [14]. Sans entrer dans les détails, on se contente de projeter toutes les fonctions, données et inconnues, sur le sous-espace de dimension finie des fonctions linéaires sur les partitions de $\Omega$ en simplexes. On aboutit alors à un problème de minimisation d'une fonctionnelle $E_{c, h}(u, v)$ (h étant la paramètre de discrétisation) en dimension finie par rapport aux deux champs $u$ et $v$. On démontre la "convergence" de $E_{c, h}(u, v)$ vers $E(u, v)$ quand $h<<k_{c}<<c \rightarrow 0$.

Pour minimiser $E_{c, h}(u, v)$ à $c, h$ constant, on ne saurait utiliser une méthode directe d'éléments finis à cause du terme $v^{2}|\nabla u|^{2}$. On se contente, sans preuve de convergence pour l'instant, de procéder à une minimisation alternée entre $u$ et $v$. On n'a alors qu'à minimiser, à chaque pas, une fonctionnelle quadratique et définie positive.

Remarque 5.3 Une méthode plus directe d'approximation de E(u) est proposée dans [16]. Les variables sont alors la triangulation $\mathbf{T}_{\mathbf{h}}$ et le champ $u ; h$ contrôle la taille de la triangulation, et $u$ est linéaire sur chacun des triangles $T \in \mathbf{T}_{\mathbf{h}}\left(u \in V_{h}(\Omega)\right)$. La fonctionnelle approchée, qui est donc définie directement sur un espace de dimension finie en $u$ est

$$
E_{h}(u, T) \equiv \begin{cases}\sum_{T \in \mathbf{T}_{\mathbf{h}}}|T \cap \Omega| \frac{1}{h_{T}} f\left(h_{T}\left(|\nabla u|^{2}\right),\right. & \text { si } u \in V_{h}(\Omega), \\ +\infty, & \text { sinon, }\end{cases}
$$

où $f$ est une fonction concave croissante sur $\mathbb{R}^{+}$telle que $f(t) / t \rightarrow 1, t \rightarrow 0$, et $f(t) \rightarrow$ $1 / 3, t \rightarrow+\infty$. La $\Gamma$-convergence vers la fonctionnelle $E(u)$ - dans un sens approprié est établie dans [16] et, une fois encore, un algorithme de minimisation par directions alternées est utilisé.

Dans le calcul numérique proprement dit, le choix des paramètres $c, k_{c}$ et du paramètre de maillage $h$ est assez délicat. De même, il convient de choisir $\Omega$ (l'ouvert de sécurité) avec circonspection. Nous renvoyons le lecteur à [14] et [17] à ce propos.

Des résultats numériques sont présentés dans [14] dans le cadre de la segmentation d’images et dans [17], Section 3, dans le cadre de la rupture antiplane.

\subsection{Le cas général}

Dans le cadre de l'élasticité bi ou tridimensionnelle, les difficultés s'amoncellent. On considère, comme dans la sous-section précédente le cas d'une densité d'énergie quadratique par rapport, cette fois, aux gradients symétrisés. Au premier pas de temps - qui sera le seul dont nous nous préoccuperons ici - le problème $\mathcal{P}_{1}$ revient alors à minimiser 


$$
\int_{\Omega \backslash \Gamma} 1 / 2 A e(u)(x) \cdot e(u)(x) d x+k \mathcal{H}^{N-1}\left(\Gamma \backslash \mathcal{N}_{1}\right)
$$

parmi tous les champs $(u, \Gamma)$ tels que $\Gamma$ (fermé $) \subset \bar{\Omega}$ et $u \in W^{1,2}(\Omega \backslash \Gamma) ; u=U_{1}$ sur $\partial \Omega_{d} \backslash \Gamma$.

Une fois encore, on voudrait introduire une formulation faible. Cependant, on ne peut plus se contenter d'une formulation sur $S B V(\Omega)$, car l'inégalité de Korn habituellement utilisée en élasticité linéarisée est en défaut dans $B V$. Il semble donc nécessaire d'introduire un nouvel espace, l'espace $S B D(\Omega)$ des éléments de $L^{1}\left(\Omega ; \mathbb{R}^{N}\right)$ dont le gradient symétrisé est une mesure. C'est malheureusement un espace plus pathologique que son analogue $S B V\left(\Omega ; \mathbb{R}^{N}\right)[9],[13]$. En particulier, on ne sait pas si le complémentaire $S_{u}$ de l'ensemble des points de LeBEsgue d'une fonction $u \in S B D(\Omega)$ n'est pas strictement plus gros que l'ensemble $J_{u}$ des sauts de $u$, i.e., des points où $u$ a une limite approchée différente à gauche et à droite (pour une direction convenablement choisie) au sens que $\mathcal{H}^{N-1}\left(S_{u} \backslash J_{u}\right)>0$; voir [9], Section 6 . On peut cependant conclure à l'existence d'une solution au problème de minimisation suivant :

Problème $\mathcal{P}_{1}$-faible : Minimiser $\int_{\Omega} A \epsilon(u) \cdot e(u) d x+k \mathcal{H}^{N-1}\left(J_{u}\right)$, pour $u \in S B V(\hat{\Omega})$ avec $u=U_{1}$ sur $\hat{\Omega} \backslash \bar{\Omega}$.

Encore faudrait-il montrer que cette formulation faible est équivalente à la formulation d'origine, ce qui reste complètement ouvert à ce jour.

On peut également entreprendre une régularisation par $\Gamma$-convergence de $\mathcal{P}_{1}$-faible dans l'esprit de la Sous-section 5.2 et l'implémenter numériquement. Quelques calculs dans ce cadre sont explicités dans [17], Section 3. Les résultats, quoique loin d'être mathématiquement justifiables sont mécaniquement très satisfaisants.

\section{Problèmes}

Cette courte section s'attache à récapituler les problèmes associés à la modélisation proposée. Pour de plus amples détails dans le cas de la rupture, nous renvoyons le lecteur à $[28]$.

Nous avons déjà évoqué en Section 4 le problème des pas de temps postérieurs au pas de temps initial dans le cas de l'endommagement. Ce problème ne semble pas se poser dans le cas de la rupture, comme on l'a vu en Section 5. En fait, la modélisation continue en temps reste une question ouverte, indépendamment de la nécessité de relaxer la fonctionnelle à un temps fixé. Même un cas d'école comme celui de la minimisation d'une fonctionnelle $\int_{\Omega} \psi(t, u(x, t)) d x$, où $\psi$ est convexe en $u$, parmi les orbites $\psi(t, u(t, x))$ décroissantes en $t$ pour tout $x$ n'est pas clair. Peut-être doit-on envisager une formulation modifiée de l'évolution.

La non unicité potentielle des solutions est troublante dans les deux contextes étudiés. Lors d'un schéma discret en temps (et d'un chargement monotone), arrive-ton au même résultat selon qu'on va directement à un temps $T$ donné ou qu'on incrémente 
le temps jusqu'à $T$ ? Les exemples numériques suggèrent l'indépendance par rapport à la discrétisation temporelle des valeurs de l'énergie totale à $T$.

Dans le cadre de la rupture, il est déraisonnable de laisser la matière s'interpénétrer. La solution apparente consiste à modifier l'énergie de surface pour interdire cette interpénétration; en d'autres termes on remplace (dans le cadre de la rupture antiplane par exemple) dans $\mathcal{P}_{i}$-faible $\mathcal{H}^{N-1}\left(S_{u} \backslash \mathcal{N}_{i}\right)$ par

$$
\int_{S_{u} \backslash \mathcal{N}_{i}} \kappa([u]) d x
$$

où

$$
\kappa([u]) \equiv\left\{\begin{array}{l}
1 \text { si }[u] \geq 0 \\
+\infty \text { sinon }
\end{array}\right.
$$

On montre aisément, par application de résultats de semi-continuité inférieure dans $S B V$ [6] l'existence d'un minimum à cette nouvelle formulation faible [15]. On ne sait cependant pas si elle est équivalente à la formulation d'origine; on ne sait pas non plus comment la "régulariser" au sens de la Sous-section 5.2.

Enfin, et c'est un obstacle de taille, on ne sait pas dans le cadre de la rupture correctement traiter des chargements par forces imposées. En effet, imposer une force, disons de volume, $f(x, t)$ revient à rajouter un terme en $-\int_{\Omega_{i}} f_{i} \cdot u d x$ dans l'énergie potentielle (discrétisée) à l'instant $t_{i}$. Mais alors 1' infimum par rapport aux champs cinématiquement admissibles $u$ devient - de manière identique dans les formulations d'origine ou faible - égal à $-\infty$ ! On peut remédier à ce désastre en introduisant la condition précédemment évoquée de non-interpénétration qui empêche, par la positivité du saut, d'envoyer $u$ à $+\infty$, mais on se convainc aisément que ceci ne convient pas si l'on applique des forces de traction sur le bord de $\Omega$. Deux voies semblent probables. Soit on ne doit pas chercher des minima globaux, ce qui a déjà été mentionné plus haut, mais les méthodes manquent, soit c'est la forme de l'énergie de surface qui est à mettre en cause, auquel cas on se résigne à abandonner la modélisation de GRIfFith.

\section{Références}

[1] E. Acerbi, N. Fusco. Semicontinuity problems in the calculus of variations. Arch. Rat. Mech. Anal., 86 1984, 125-145.

[2] G. Altaire. Explicit lamination parameters for three-dimensional shape optimization. Control and Cybernetics, 23, 1994, 309-326.

[3] G. Allaire, S. Aubry, F. Jouve. Simulation numérique de l'endommagement à l'aide du modèle Francfort-Marigo. ESAIM Proceedings, 3, 1998, 1-9.

[4] G. Allaire, R.V. Kohn. Optimal bounds on the effective behavior of a mixture of two well-ordered elastic materials. Quart. Appl. Maths., LI-4, 1993, 643-674.

[5] L. Ambrosio. Variational problems in $S B V$ and image segmentation. Acta Appl. Math., 17, $1989,1-40$. 
[6] L. Ambrosio. Existence theory for a new class of variational problems. Arch. Rat. Mech. Anal., 111-1, 1990, 291-322.

[7] L. Ambrosio, V.M. Tortorelli. Approximation of functionals depending on jumps by elliptic functionals via $\Gamma$-convergence. Comm. Pure Appl. Math., XLIII, 1990, 999-1036.

[8] L. Ambrosio, V.M. Tortorelli. On the approximation of free discontinuity problems. Boll. Un. Mat. Ital. ,7-6B, 1992, 105-123.

[9] L. Ambrosio, A. Coscia, G. Dal Maso. Fine properties of functions with bounded deformations. A paraître.

[10] M. Amestoy. Propagations de fissures en élasticité plane. Thèse d'Etat, 1987, Paris.

[11] J.M. Ball, F. Murat. $W^{1, p}$-quasiconvexity and variational problems for multiple integrals. J. Funct. Anal., 58, 1984, 225-253.

[12] G. Bellettini, A. Coscia. Discrete approximation of a free discontinuity problem. Numer. Funct. Anal. Optim, 15, 1994, 105-123.

[13] G. Bellettini, A. Coscia, G. Dal Maso. Special functions of bounded variations. A paraître.

[14] B. Bourdin. Image segmentation with a finite element method. RAIRO Modél. Math. Anal. Numér., 1998. A paraître.

[15] B. Bourdin. Une formulation variationnelle en mécanique de la rupture, théorie et applications numériques. Thèse de Doctorat, 1998, Université Paris Nord.

[16] B. Bourdin, A. Chambolte. Implementation of an adaptive finite element approximation of the Mumford-Shah functional. A paraitre.

[17] B. Bourdin, G.A. Francfort, J.-J. Marigo. Numerical experiments in revisited brittle fracture. A paraitre.

[18] A. Braides, G. Dal Maso, A. Garoni,. Variational Formulation of softening phenomena in fracture mechanics : the one-dimensional case. A paraitre.

[19] H.D. Bui. Mécanique de la rupture fragile. Masson,1978, Paris.

[20] M. Carriero, A. Leaci. Existence theorem for a Dirichlet problem with free discontinuity set. Nonlinear Anal., Th. Meth. Appls., 15-7, 1990, 661-677.

[21] A. Chambolle, G. Dal Maso. Discrete approximation of the Mumford-Shah functional in dimension two. RAIRO Modél. Math. Anal. Numér., 1998. A paraître.

[22] A.V. Cherkaev, L.V. Gibiansky. Microstructures of composites of extremal rigidity and exact bounds on the associated energy density. In A. Cherkaev, R. Kohn, editors, Topics in the mathematical modelling of composite materials, Progress in Nonlinear Differential Equations and Their Applications, Birkhäuser, Boston, 1997, 273-317.

[23] E. De Giorgi, M. Carriero, A. Leaci. Existence theorem for a minimum problem with free discontinuity set. Arch. Rat. Mech. Anal., 108, 1989, 195-218.

[24] L.C. Evans, G.F. Gariepy. Measure theory and fine properties of functions. CRC Press, 1992, Boca Raton.

[25] I. Fonseca, G.A. Francfort. Relaxation in BV versus quasiconvexification in $W^{1, p}$; a model for the interaction between fracture and damage. Calc. Var., 3-4, 1995, 407-446. 
[26] I. Fonseca, S. Muller. A-quasiconvexity, lower semi-continuity and Young measures. A paraître.

[27] G.A. Francfort, J.-J. Marigo. Stable damage evolution in a brittle continuous medium. Eur. J. Mech. A/Solids, 12-2, 1993, 149-189.

[28] G. Francfort, J.-J. Marigo. Revisiting brittle fracture as an energy minimization problem. J. Mech. Phys. Solids, 46-8, 1998, 1319-1342.

[29] G. Francfort, J.-J. Marigo. Cracks in fracture mechanics : a time- indexed family of energy minimizers. In P. Argoul, M. Frémond, Q.S. Nguyen, editors, IUTAM Symposium on Variations of domains and free-boundary problems in solid mechanics, Solid Mechanics and its Applications, Vol. 66, Kluwer, Dordrecht, 1999, 197-203.

[30] A. Griffith. The phenomena of rupture and flow in solids. Phil. Trans. Roy. Soc. London, CCXXI-A, 1920, 163-198.

[31] Z. HASHIN. Finite thermoelastic fracture criterion with application to laminate cracking analysis. J. Mech. Phys. Solids,44-7, , 1996, 1129-1145.

[32] R.V. Konn. The relaxation of a double-well energy. Cont. Mech. Thermodyn., 3, 1991, $193-236$

[33] J. Lemaitre, J.L. Chaboche. Mécanique des matériaux solides. Dunod, 1985, Paris.

[34] F. LÉNÉ. Contribution à l'etude des matériaux composites et de leur endommagement. Thèse d'état,1984, Paris.

[35] P. Marcellini. Periodic solutions ans homogenisation of nonlinear variational problems. Ann. Mat. Pura Appl., 1178, 1978, 139-152.

[36] D. Mumford, J. Shah. Optimal approximation by piecewise smooth functions and associated variational problems. Comm. Pure Appl. Math., 42, 1989, 577-685.

[37] S. MulLeR. Homogenization of nonconvex integral functionals and cellular elastic materials. Arch. Rat. Mech. Anal., 99, 1987, 189-212.

[38] Q.S. NGUYEN. Bifurcation and post-bifurcation analysis in plasticity and brittle fracture. $J$. Mech. Phys. Solids,35, 1987, 303-324.

[39] J.M. Rossi. Application de méthodes d'homogénéisation à l'étude numérique de problèmes d'optimisation de formes. Thèse de l'université de Provence, 1996, Marseille.

[40] L. Truskinovsky. Fracture as a phase transition. In R. C. Batra and M. F. Beatty, editors, Contemporary research in the Mechanics and Mathematics of Materials, CIMNE, 1996, Barcelona. 